\title{
Inflammatory Cytokines Induce Podoplanin Expression at the Tumor Invasive Front
}

Akiko Kunita, ${ }^{* \dagger}$ Vanessa Baeriswyl, ${ }^{\star}$ Claudia Meda, ${ }^{\dagger}$ Erik Cabuy, ${ }^{\S \Phi}$ Kimiko Takeshita, ${ }^{\dagger}$ Enrico Giraudo, ${ }^{\ddagger}$ Andreas Wicki, ${ }^{*}$ Masashi Fukayama, ${ }^{\dagger}$ and Gerhard Christofori*

From the Department of Biomedicine, * University of Basel, Basel, Switzerland; the Department of Pathology, ${ }^{\dagger}$ Graduate School of Medicine, The University of Tokyo, Tokyo, Japan; the Laboratory of Transgenic Mouse Models, ${ }^{\ddagger}$ Candiolo Cancer Institute-The Fondazione del Piemonte per l'Oncologia, Istituto di Ricovero e Cura a Carattere Scientifico (IRCCS), Torino, Italy; the Department of Science and Drug Technology, University of Torino, Candiolo, Italy; the Friedrich Miescher Institute for Biomedical Research, ${ }^{\S}$ Basel, Switzerland; and The CAEX Project, ${ }^{\Uparrow}$ CAEX NV, Lier, Belgium

Accepted for publication January 23, 2018.

Address correspondence to Gerhard Christofori, Ph.D., Department of Biomedicine, University of Basel, Mattenstrasse 28, CH-4058 Basel, Switzerland. E-mail: gerhard. christofori@unibas.ch.

\begin{abstract}
Tumor invasion is a critical first step in the organismic dissemination of cancer cells and the formation of metastasis in distant organs, the most important prognostic factor and the actual cause of death in most of the cancer patients. We report herein that the cell surface protein podoplanin (PDPN), a potent inducer of cancer cell invasion, is conspicuously expressed by the invasive front of squamous cell carcinomas (SCCs) of the cervix in patients and in the transgenic human papillomavirus/estrogen mouse model of cervical cancer. Laser capture microscopy combined with gene expression profiling reveals that the expression of interferon-responsive genes is up-regulated in PDPN-expressing cells at the tumor invasive front, which are exposed to CD45-positive inflammatory cells. Indeed, PDPN expression can be induced in cultured SCC cell lines by single or combined treatments with interferon$\gamma$, transforming growth factor- $\beta$, and/or tumor necrosis factor- $\alpha$. Notably, shRNA-mediated ablation of either PDPN or STAT1 in A431 SCC cells repressed cancer cell invasion on s.c. transplantation into immunodeficient mice. The results highlight the induction of tumor cell invasion by the inflammatory cytokine-stimulated expression of PDPN in the outermost cell layers of cervical SCC. (Am J Pathol 2018, 188: 1276-1288; https://doi.org/10.1016/j.ajpath.2018.01.016)
\end{abstract}

Tumor cell migration and tissue invasion is a critical process underlying the intravasation of malignant cancer cells into the blood or lymphatic stream and their dissemination to distant organs to form metastases, the primary cause of mortality in cancer patients. Tumor invasion is often distinguished into single-cell migration induced by an epithelial-to-mesenchymal transition, an amoeboid type of migration involving the loss of integrin-mediated cell-matrix adhesion and of protease activities, and into collective invasion in which cancer cells migrate as clusters of tumor cells into the surrounding tissue. ${ }^{1-3}$ Podoplanin (PDPN) has been recently identified as a major marker for invasive cancer cells in several cancer subtypes, including malignant brain tumors, ${ }^{4}$ mesotheliomas, ${ }^{5}$ osteosarcomas, ${ }^{6}$ and, most abundantly, squamous cell carcinomas (SCCs). ${ }^{7-10}$

PDPN is a small membrane glycoprotein with an extracellular domain rich in O-glycoside side chains, a transmembrane domain, and a short nine amino acid intracellular domain that, via three basic amino acids, binds to the ERM family proteins ezrin, radixin, and moesin. ${ }^{11,12}$ On the extracellular side, three adjacent tandem repeats have been identified, O-glycan on the third of which (platelet aggregation-stimulating domain 3) binds to the PDPN receptor C-type-lectin-like-2 on platelets. ${ }^{13,14}$ PDPN is expressed in many cell types of normal tissue, including lymphatic endothelial cells, type I pneumocytes, glomerular podocytes, and many more. ${ }^{10}$ PDPN-deficient mice die at birth because of respiratory failure caused by an

\footnotetext{
Supported by Japan Society for the Promotion of Science Grants-in-Aid for Scientific Research grant 23890036 (A.K.), Italian Association for Cancer Research L' Associazione Italiana per la Ricerca sul CancroInvestigator Grant 15645 (E.G.), the Swiss National Science Foundation grant 31003A-144206 (G.C. and A.W.), the Swiss Cancer League grant OCS-02102-08-2007 (G.C. and A.W.), and the Krebsliga Beider Basel grant 04-2010 (G.C. and A.W.).

A.K. and V.B. contributed equally to this work.

Disclosures: None declared.
} 
underdevelopment of pulmonary alveoli, yet also exhibiting dilated malfunctioning lymphatic vessels and lymphedema. ${ }^{15}$ Notably, PDPN-mediated platelet aggregation on the surface of PDPN-expressing lymphatic sacs has been found critical for the separation of the lymphatic from the blood vascular system. ${ }^{16}$ On the other hand, PDPN expression on keratinocytes has been implicated in wound healing and the development of psoriasis. ${ }^{17,18}$ In addition, many more physiological and pathophysiological functions have been attributed to PDPN's expression in a variety of cell types. ${ }^{10,19}$

Despite its expression in many cell types of normal tissue, PDPN is frequently used as a diagnostic marker in cancer patients. In most of these cases, PDPN expression demarks the fronts of cancer cells collectively invading cancer into the surrounding tissue either in the absence of an epithelial-tomesenchymal transition or in the presence of an epithelial-tomesenchymal transition. ${ }^{7,9,20,21}$ Its expression seems to be regulated by various signaling pathways and transcription factors, including transforming growth factor (TGF)$\beta-\mathrm{SMAD}$ and epidermal growth factor receptor-STAT1/3 signaling and by prospero homeobox 1 and AP-1 transcription factor activities. ${ }^{6,22,23}$ PDPN expression correlates with tumor cell invasiveness in vitro and in vivo. For example, the forced expression of PDPN in a variety of differentiated cancer cells has led to the formation of filopodia and increased cell migration and invasion in vitro, and the transgenic expression of PDPN in mouse models of cancer has led to collective tumor cell invasion in vivo. ${ }^{9,11,24}$ However, in many cancer types, including breast, kidney, liver, and several others, PDPN is exclusively expressed by cancer-associated fibroblasts and correlates with malignant cancer progression. ${ }^{25,26}$

How PDPN transmits a promigratory signal to cancer cells is only poorly understood. Although it has been shown that its interaction with ezrin, radixin, and moesin proteins and the subsequent regulation of RhoA GTPase activity is critical for actin cytoskeleton reorganization and cell migration, ${ }^{9,11}$ the actual mode of signal transduction remains unclear. An interaction with the stem cell and metastasis-associated surface marker CD44 seems to be critical for directed cancer cell migration. ${ }^{27}$ Other interactions (eg, with galectin- 8 ) could be important. ${ }^{28}$ Serine phosphorylation of the intracellular domain of PDPN by protein kinase A has been shown to repress cell motility. ${ }^{29}$

Consistent with the maintenance of a differentiated phenotype in collective cancer cell invasion, high PDPN expression has been shown as a prognostic marker for good clinical outcome and lower frequency of metastasis formation. ${ }^{30,31}$ On the other hand, by binding to its receptor $\mathrm{C}$-type-lectin-like-2 present on platelets, PDPN-expressing cancer cells aggregate with platelets to protect them from immune surveillance in the blood stream, thus facilitating tumor metastasis. ${ }^{32-34}$

Although the ability of PDPN to induce tumor cell invasion and migration is generally appreciated, the mechanisms of how the distinct expression of PDPN in the outermost cell layer of the invasive tumor front is induced and how PDPN expression leads to increased invasiveness of SCC are still poorly understood. Using gene expression profiling and subsequent functional experimentation, we herein report that the expression of PDPN in the invasive front of SCC is induced by inflammatory cytokines, including interferon- $\gamma$, TGF- $\beta$, and tumor necrosis factor- $\alpha$ and their downstream STAT1 and SMAD-dependent signaling pathways. High PDPN expression then leads to SCC cell migration and invasion. Accordingly, ablation of STAT1 or PDPN expression results in a reduction of SCC invasion in a transplantation mouse model of SCC in vivo.

\section{Materials and Methods}

\section{Cells and Tissue Samples}

Neonatal human epidermal keratinocytes (KURABO, Osaka, Japan) were cultured in HuMedia-KG2 serum-free medium (KURABO). Human SCC cell lines, SCC9, A431, and $\mathrm{FaDu}$, were grown in Dulbecco's modified Eagle's medium (Sigma Chemical Co, St. Louis, MO), and H226 was cultured in RPMI 1640 medium (Sigma Chemical Co) with 10\% fetal bovine serum (CELLect; MP Biomedicals, Irvine, CA), $100 \mathrm{U} / \mathrm{mL}$ penicillin, and $100 \mu \mathrm{g} / \mathrm{mL}$ streptomycin (Sigma Chemical Co), at $37^{\circ} \mathrm{C}$ saturated with $5 \%$ $\mathrm{CO}_{2}$ in a humid atmosphere.

Ten cases of invasive squamous cell carcinomas (International Federation of Gynecology and Obstetrics stage IIb) of the cervix were obtained during surgery and retrieved from the files of the University of Tokyo Hospital (Tokyo, Japan) during 1999 to 2007. This study was approved by the Ethics Committee of the Graduate School of Medicine of the University of Tokyo (number 2381).

\section{Animals}

Female BALB/c nude (nu/nu) mice (5 weeks old) were purchased from Charles River Japan, Inc. (Kanagawa, Japan). Keratin-14 (K14)-human papillomavirus serotype 16 (HPV16)/estrogen $\left(\mathrm{E}_{2}\right)$ transgenic mice have been described elsewhere. ${ }^{35}$ To generate A431 xenograft samples, $10^{6}$ cells were intradermally injected into the back of nude mice, and the mice were sacrificed after 17 days. Mice were handled under specific-pathogen-free conditions in accordance with the policies of the Animal Ethics Committee of The University of Tokyo (P16-080).

\section{Laser Microdissection}

Sections $(14 \mu \mathrm{m}$ thick) from frozen mouse tissues $(N=4$ mice) were prepared using a cryostat, placed on membrane slides (MMI, Glattbrugg, Switzerland), fixed for 1 minute in $70 \%$ ethanol, and rinsed in water for a few seconds. They were then stained with Mayer's hematoxylin for 30 seconds and then rinsed for 10 seconds in water, 30 seconds in $70 \%$ ethanol, and 30 seconds in 95\% ethanol. The sections were 
allowed to dry, and then cells of interest (invasive tumor front, tumor mass, and normal epithelium) were dissected using an MMI CellCut laser microdissection microscope (Molecular Machines \& Industries $\mathrm{GmbH}$, Eching, Germany). Approximately 100 cells were pooled from a specific layer of interest and used for RNA extraction. Eighty-one samples were taken in total, and six samples per condition (PDPN-positive tumor cells, PDPN-negative tumor cells, and normal epithelium) were further analyzed by gene expression profiling on the basis of tissue morphology and PDPN immunostaining.

\section{RNA Isolation, Amplification, and Microarray Profiling}

Laser-dissected cells (12 samples) were immediately covered with $50 \mu \mathrm{L}$ extraction buffer (Arcturus Bioscience, Mountain View, CA), left to lyse on the lid of the tube at room temperature for at least 15 minutes, and then stored at $-80^{\circ} \mathrm{C}$. Total cellular RNA was isolated with the PicoPure RNA isolation kit (Arcturus Bioscience), with minor modifications. The optional DNase treatment step to remove potential genomic DNA contamination was omitted, and the centrifugation times during washing were reduced to 30 seconds. All RNA samples were processed in batches of maximally four times three replicates (12 samples in total). Preparation of in vitro transcription products was performed according to Affymetrix protocols (GeneChip Expression Analysis Technical manual, revision 5), with minor modifications. The fragmented cRNA $(15 \mu \mathrm{g})$ was used in a hybridization cocktail containing spiked controls (Affymetrix, Santa Clara, CA). A total of $200 \mu \mathrm{L}$ of this hybridization cocktail was hybridized at $45^{\circ} \mathrm{C}$ for 16 hours to GeneChip Mouse Genome 4302.0 Arrays (Affymetrix). After hybridization, the arrays were processed using a GeneChip Fluidics Station 400, according to recommended protocols (FS450_0001; Affymetrix). Fluorescent images of arrays were captured using the GeneChip Scanner 30007 G (Affymetrix), and image data were acquired and analyzed using the GeneChip Operating Software (Affymetrix). Average present call values were approximately $50 \%$.

\section{Microarray Data Analysis}

Expression values and detection $P$ values from the microarray data were calculated using the GC-RMA implementation with the Affymetrix Expression Console software version 4.0. Gene expression changes were evaluated on removal of any gene with a detection of $P>0.04$ in all conditions. Gene expression profiles were compared using GeneSpring Software version 7.3 (Silicon Genetics, Redwood City, CA) and Simple Array Analyzer (iAnalyze, Kagoshima, Japan). A statistical power analysis was performed to determine the minimum fold difference with a power of 0.8 or better. On the basis of this, our experiment was able to reliably detect fold changes $>1.5$. To further reduce the number of genes in the analysis, either a 1.5- or 2-fold filter was performed on the genes present in at least one condition, and union list of these genes was generated (this reduced the false discovery implications of subsequent statistical analyses). This list was then used to identify statistically significant effects in the data set using an $\mathrm{N}$-way analysis of variance $(P<0.01)$. False discovery rate was estimated using a Benjamini and Hochberg correction. Genes passing these filters were then subjected to gene enrichment analysis using GENECODIS, a web-based tool for enrichment analysis (GeneCodis, http://genecodis. cnb.csic.es, last accessed December 23, 2017) using Gene Ontology Biological Process. ${ }^{36-38}$ All gene expression data are available at Gene Expression Omnibus at $h t t p s: / / w w w$. ncbi.nlm.nih.gov/geo (accession number GSE108208).

\section{Real-Time PCR Analysis}

The amplified cRNA $(0.25 \mu \mathrm{g})$ generated from laserdissected cells was reverse transcribed for first-strand cDNA synthesis for 50 minutes at $50^{\circ} \mathrm{C}$ using Superscript III (Life Technologies, Carlsbad, CA) with oligo $\mathrm{dT}_{12-18}$ primers (Life Technologies). The samples were then heated for 15 minutes at $70^{\circ} \mathrm{C}$ to inactivate the reverse transcriptase. Total RNA from cell lines was prepared using ISOGEN II (Nippon Gene Co Ltd, Toyama, Japan). Total RNA $(0.5 \mu \mathrm{g})$ was used for the subsequent synthesis of cDNA using the ReverTra Ace qPCR RT Kit (Toyobo Co Ltd, Osaka, Japan), as recommended by the manufacturer. The mRNA levels were measured using an ECO Real-Time PCR system (Illumina Inc., San Diego, CA) and KAPA SYBR Fast qPCR Kit (Kapa Biosystems Inc., Woburn, MA) with the following primer pairs: mouse PDPN, 5'-GCCAACTTGTAACTGCACTTGAT-3' (forward) and $5^{\prime}$-GAAGGCACAGTGGATGCTTAG-3' (reverse); mouse glyceraldehyde-3-phosphate dehydrogenase, 5'-AGCTTGTCATCAACGGGAAG-3' (forward) and 5'-TTTGATGTTAGTGGG GTCTCG-3' (reverse); human PDPN, 5'-CCAGCGAAGACCGCTATAAGTC-3' (forward) and 5'-CACTTGTTGCCACCAGAGTTGT-3' (reverse); human glyceraldehyde3-phosphate dehydrogenase, 5'-GAAGGTGAAGGTCGGAGTC- $3^{\prime}$ (forward) and $5^{\prime}$-GAAGATGGTGATGGGATTC- $3^{\prime}$ (reverse). All PCRs were performed in a $10 \mu \mathrm{L}$ volume, using 48-well PCR plates (Illumina). The cycling conditions were $95^{\circ} \mathrm{C}$ for 1 minute (enzyme activation), followed by 45 cycles of $95^{\circ} \mathrm{C}$ for 5 seconds and $58^{\circ} \mathrm{C}$ for 30 seconds. After amplification, the samples were heated slowly from $55^{\circ} \mathrm{C}$ to $95^{\circ} \mathrm{C}$ with continuous reading of fluorescence to obtain a melting curve. Relative mRNA levels were calculated by the difference between the threshold cycle of a given target cDNA and that of an endogenous reference cDNA $\left(\Delta \mathrm{C}_{\mathrm{T}}\right)$.

\section{Immunofluorescence Analysis and} Immunohistochemistry

For immunofluorescence staining, cells grown on coverslips were fixed with $4 \%$ paraformaldehyde in phosphate-buffered 


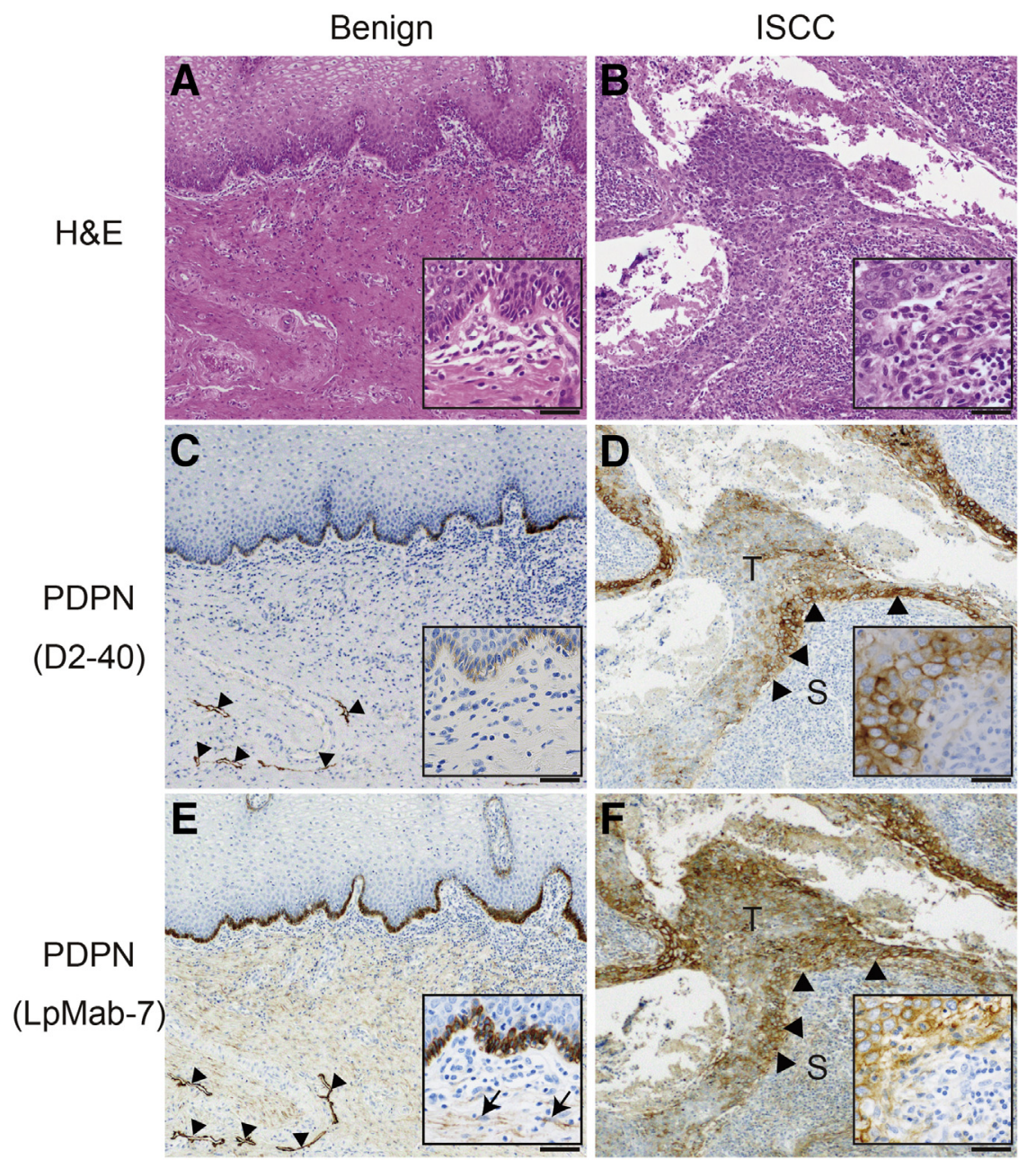

Figure 1 PDPN expression at the invasive front of human cervix squamous cell carcinoma (SCC). Three serial paraffin sections from an SCC of a patient were processed for staining with hematoxylin and eosin (H\&E; A and B) or for PDPN immunohistochemistry using anti-PDPN antibodies D2-40 (C and D) or LpMab-7 (E and $\mathbf{F}$ ). In benign cervical squamous epithelium, weak PDPN expression is detected in the basal cell layer. C: Arrowheads indicate lymphatic vessels. D and F: Intense PDPN expression is seen in tumor cells ( $T$ ) at the tumor invasive front (arrowheads) of invasive SCC (ISCC), adjacent to the tumor stroma (S). E: PDPN expression in the stromal cells was detected only by using LpMab-7 antibody (inset). Arrowheads indicate lymphatic vessels; arrows, stromal cells. Insets: Magnification of area of interest. Scale bar $=100 \mu \mathrm{m}(\mathbf{A}-\mathbf{F})$. Original magnification, $\times 400$ (insets).

saline for 15 minutes. Fixed cells were permeabilized with $0.2 \%$ Triton X-100 in phosphate-buffered saline for 5 minutes, blocked with $1 \%$ bovine serum albumin in phosphate-buffered saline for 30 minutes, and incubated with the following: anti-mouse PDPN antibody (clone 8.1.1), anti-E-cadherin antibody (610182; BD Transduction Laboratories, Franklin Lakes, NJ), anti-phosphorylated (phospho)-STAT3 (9167; Cell Signaling Technology Inc., Danvers, MD), or antiphospho-SMAD2 (3101; Cell Signaling Technology Inc.) for 1 hour. This was followed by incubation with Alexa Fluor 488- and Alexa Fluor 546-conjugated secondary antibody (Life Technologies) for 1 hour.

Multiplexed immunofluorescence was performed using the Opal Multiplex Immunohistochemistry Kit (Perkin Elmer, Norwalk, CT). After deparaffinization, heat-mediated antigen retrieval was performed. The primary antibodies included anti-mouse PDPN (8F11 $\left.{ }^{33} ; 1: 1000\right)$, anti-CD45 (30-F11; 1:500; BD Biosciences, Franklin Lakes, NJ), anti-CD4 (GK1.5; 1:250; Thermo Fisher Scientific, Fremont, CA), and F4/80 (D2S9R; 1:500; Cell Signaling Technology Inc.). Primary antibodies were incubated for 30 minutes at room temperature. Opal Polymer horseradish peroxidase or anti-rat secondary antibody (MP-7444-15; Vector Laboratories,
Burlingame, CA) was added and incubated for 10 minutes. Opal fluorophores were used to detect fluorescent signal, with the opal 520 used for PDPN, the opal 570 used for CD45, the opal 620 used for CD4, and the opal 690 used for F4/80. Images were obtained on the Mantra Quantitative Pathology Imaging microscope version 1.0.3 and analyzed using inform software version 2.3.0 (both from Perkin Elmer).

For immunohistochemistry of human tissue samples, formalin-fixed, paraffin-embedded sections were heat treated with $10 \mathrm{mmol} / \mathrm{L}$ sodium citrate buffer $(\mathrm{pH}$ 6.0) and immunostained using anti-PDPN (D2-40; AngioBio, Del Mar, CA; or clone LpMab- ${ }^{39}$ ), anti-phospho-STAT1 (Tyr701; 9167; Cell Signaling Technology Inc.), or anti-phospho-STAT3 (9145; Cell Signaling Technology Inc.).

\section{Immunoblotting}

Whole cell lysates from cultured cells were prepared and separated using SDS-PAGE and immunoblotted, as previously described. ${ }^{40}$ The membranes were incubated with antibodies against PDPN (clone NZ-1 ${ }^{41}$ ), phospho-SMAD2 (3101; Cell Signaling Technology Inc.), SMAD2 (3103; Cell Signaling Technology Inc.), phospho-STAT1 (9167; 


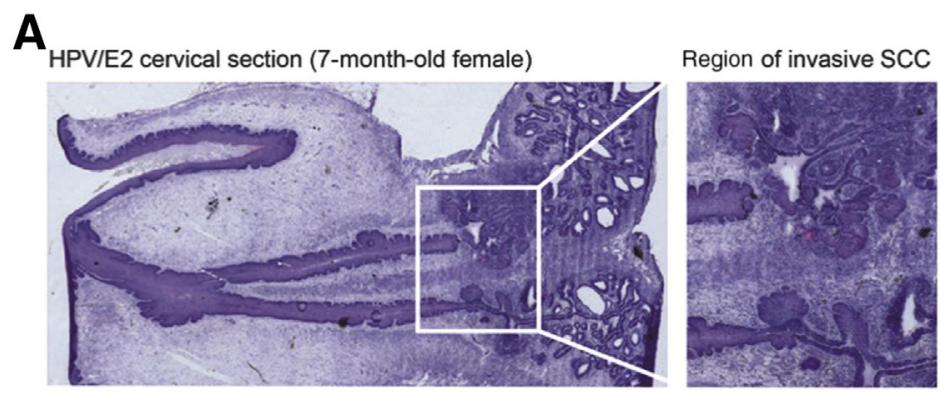

B
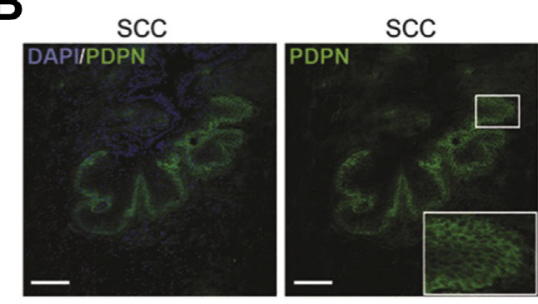
Normal epithelium
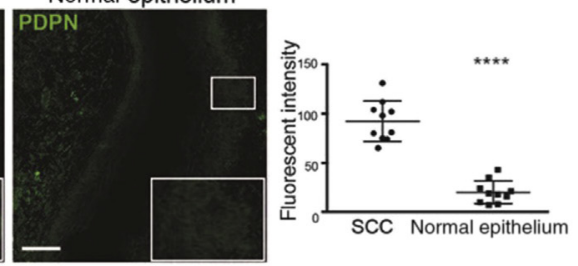

c
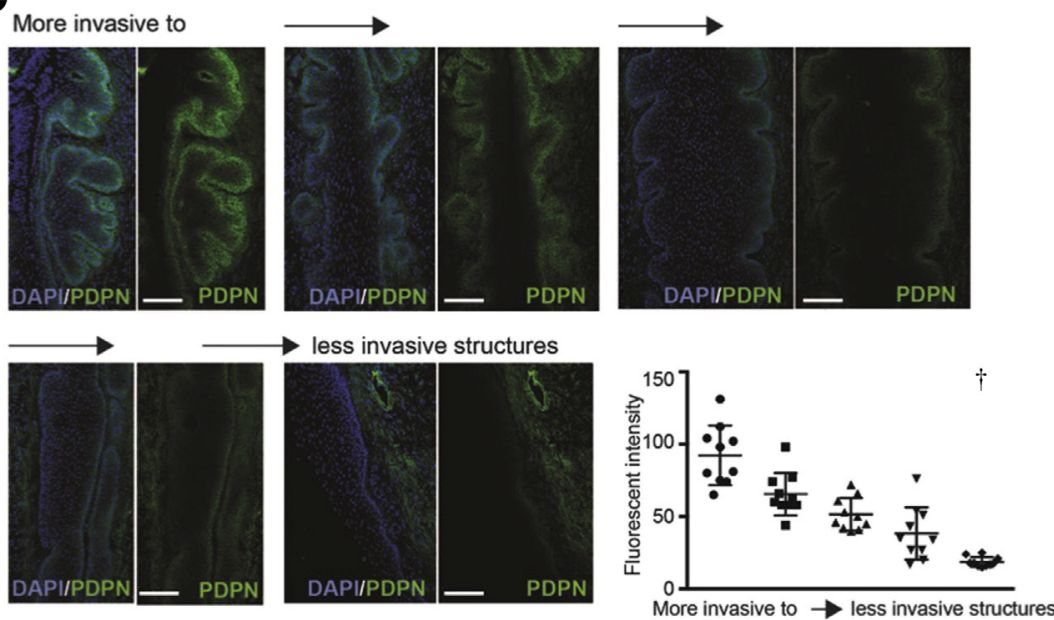

Figure 2 PDPN expression at the invasive front of squamous cell carcinoma (SCC) of the cervix in human papillomavirus (HPV)/estrogen $\left(\mathrm{E}_{2}\right)$ transgenic mice. A: Hematoxylin staining of histologic sections of the cervix and upper vagina of a keratin-14-HPV16 mouse. Invasive SCC localizes to the cervical transformation zone. B: Immunofluorescence staining of PDPN (green) in a squamous carcinoma region and in normal epithelium. PDPN staining was detected in SCC, but not in the normal epithelium. DAPI (blue) was used to visualize cell nuclei. Fluorescence staining intensity of PDPN in SCC is significantly higher than in normal epithelium (right panel). A merged image of DAPI and PDPN staining of the middle panel is shown in the left panel. Boxed areas are shown at higher magnification in the insets. C: PDPN expression (green) is high in the invasive tumor areas and low in the noninvasive regions of SCC. DAPI (blue) was used to visualize cell nuclei. Same magnification as B. Fluorescence staining intensity of PDPN in SCC significantly correlates with the higher degree of tumor invasiveness (right bottom panel; Spearman $r=-1.000) . n=10$ sections each (B); $n=6$ tumors and $9-10$ sections of each tumor stage (C). ${ }^{* * * * P}>0.0001$ versus SCC; ${ }^{\dagger} P<0.05$ versus high invasive structures. Scale bars $=100$ $\mu \mathrm{m}(\mathbf{B}$ and $\mathbf{C})$. Original magnification, $\times 20(\mathbf{A})$.
Cell Signaling Technology Inc.), STAT1 (9172; Cell Signaling Technology Inc.), and $\beta$-actin (A5441; SigmaAldrich Corp., St. Louis, MO).

\section{Statistical Analysis}

Data sets besides microarray data were analyzed using Graphpad Prism version 4 (Graphpad Software Inc., San Diego, CA) and shown as means \pm SEM. $U$-test or one-way analysis of variance, followed by Tukey-Kramer multiple comparisons, was performed where appropriate. $P<0.05$ was considered statistically significant.

\section{Results}

PDPN Expression at the Invasive Front of Cervix Squamous Carcinoma

PDPN has been previously reported to be specifically expressed in the outermost cell layer of the invasive front of
SCCs of various tissues and organs. ${ }^{9}$ Motivated by these observations, the cellular and molecular mechanisms underlying such defined pattern of PDPN expression were studied. First, the expression pattern of PDPN was studied using two kinds of anti-PDPN antibodies (routinely used D2- $40^{42}$ and newly established LpMab- $7^{39}$ ) in human cervix SCC by immunohistochemistry (Figure 1). In benign cervical squamous epithelia, substantial PDPN expression was detected by both antibodies exclusively in the outermost cell layer of finger-like protrusions and was predominantly located at the plasma membrane. LpMab-7 detected PDPN expression more concisely in the epithelial cells and in the lymphatic endothelium (Figure 1, A, C, and E). Moreover, LpMab-7 visualized PDPN on $\alpha$-smooth muscle actin-positive stromal fibroblasts (Figure 1E and Supplemental Figure S1). On the other hand, in invasive squamous cell carcinoma, high PDPN expression was mainly restricted to the periphery of the invading tumor fronts, which still appeared to collectively invade into the neighboring organ parenchyma. In contrast, PDPN expression was absent in the center of the tumors (Figure 1, B, D, and F). 

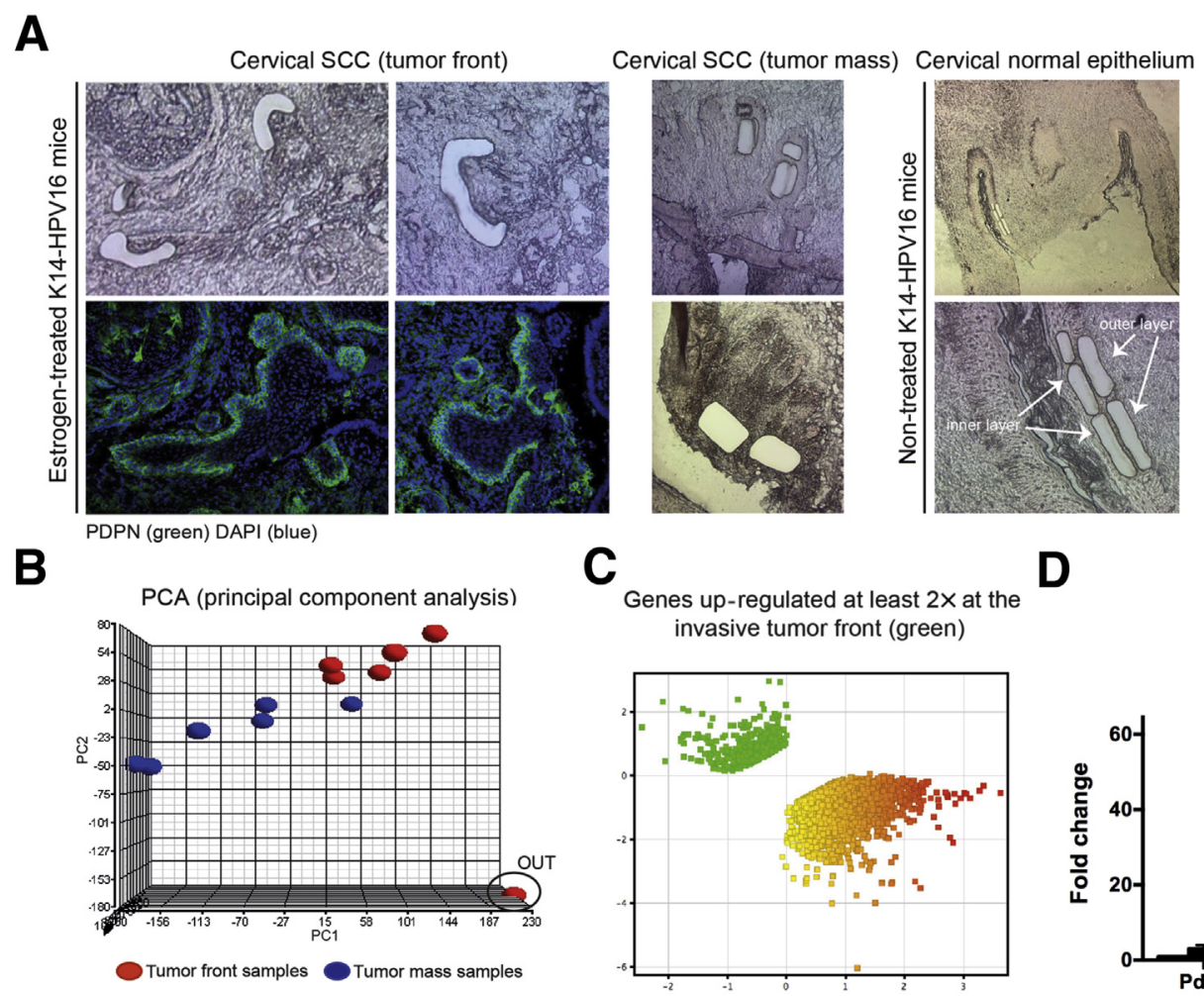
invasive tumor front (green)
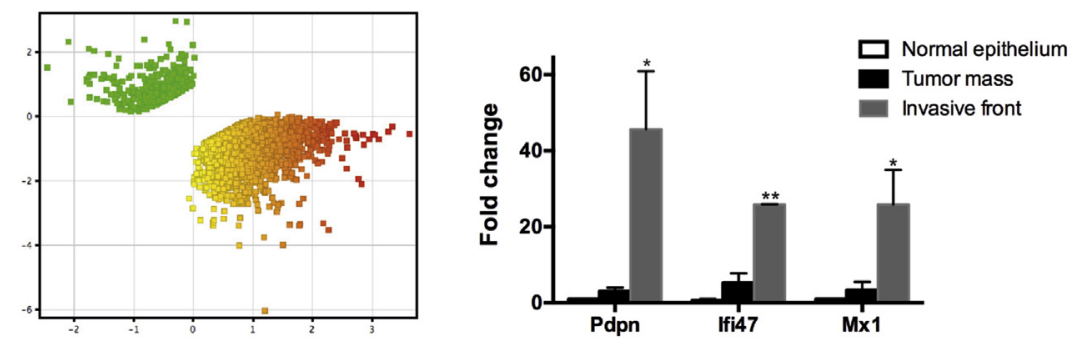

Figure 3 Up-regulation of interferon-response genes at the invasive front of cervical squamous cell carcinoma (SCC) in human papillomavirus (HPV)/ estrogen $\left(E_{2}\right)$ mice. A: Frozen sections of cervical SCC of HPV/E $E_{2}$ mice were stained with hematoxylin, and microdissected into invasive front, tumor mass, and normal epithelium. Holes in the section indicate the area of tissue isolation by laser capture microscopy. Immunofluorescence staining of PDPN (green) on serial sections is shown below. B: Principal component analysis (PCA) of gene expression in the dissected invasive front and the inner tumor mass. An outlying sample of the tumor front was removed for further analysis. C: A total of 209 genes are significantly up-regulated at least twofold at the tumor front (green) compared with the inner tumor mass. The list of the genes is detailed in Supplemental Table S1. D: Validation of the up-regulated expression of PDPN, Ifi47, and $\mathrm{Mx1}$ in the tumor invasive front by quantitative RT-PCR. ${ }^{*} P<0.05,{ }^{*} P<0.01$ versus tumor mass. Original magnification, $\times 200($ A). K14, keratin-14.

To investigate the peculiar PDPN expression pattern during the multiple stages of SCC progression, $\mathrm{HPV} / \mathrm{E}_{2}$ mouse model of cervix $\mathrm{SCC}^{43}$ was used. K14-HPV16 mice express part of the HPV16 genome under the control of the K14 promoter, leading to the expression of the HPV16 E6 and E7 oncogenes in the basal cells of squamous epithelia. ${ }^{44}$ The mice subsequently develop progressive epidermal neoplasia, starting with hyperplastic lesions from which dysplasia appears with age. When backcrossed into the FBV/n background, susceptible to epidermal malignancy, SCC develops from dysplastic lesions. Chronic estrogen treatment via implantation of $17 \beta$-estradiol $\left(\mathrm{E}_{2}\right)$ capsules in K14-HPV16 females $\left(\mathrm{HPV} / \mathrm{E}_{2}\right)$ sustains the estrus phase and leads, in a multistage manner, to invasive uterine cervical

Table 1 Consistently Enriched G0 at the Invasive Tumor Front

\begin{tabular}{|c|c|c|c|}
\hline G0 identification & Category & $P$ value* & Up-regulated genes \\
\hline 0060337 & Type I interferon-mediated signaling pathway & $1.57 \times 10^{-7}$ & Usp18, Ifi35, Oas2, Gbp2, Irf7, Ifit1, Mx1 \\
\hline 0019221 & Cytokine-mediated signaling pathway & & \\
\hline 0043627 & Response to estrogen stimulus & $1.47 \times 10^{-6}$ & Bcl2, Cond1, Brca1 \\
\hline 0045087 & Innate immune response & $3.51 \times 10^{-6}$ & Vnn1, Nfkbia, Bcl2, Irak1 \\
\hline 0006916 & Antiapoptosis & & \\
\hline 0006260 & DNA replication & $1.82 \times 10^{-5}$ & Trex1, Pold1, Set, Brca1, Nfic, Mcm7, Igf1 \\
\hline 0008283 & Cell proliferation & $2.84 \times 10^{-4}$ & Fscn1, Bcat1, Crip1, Pa2g4, Pdpn, Tspo, Tpx2, Mcm7 \\
\hline 0060333 & Interferon- $\gamma-$ mediated signaling pathway & $1.09 \times 10^{-3}$ & Oas2, Gbp2, Irf7 \\
\hline 0000278 & Mitotic cell cycle & $1.27 \times 10^{-3}$ & Pmf1, Sfi1, Cond1, Pold1, Tubgcp5, Incenp, Mcm7 \\
\hline 0016477 & Cell migration & $1.87 \times 10^{-2}$ & Fscn1, Nanos1, Dgkz \\
\hline
\end{tabular}

*Hypergeometric $P$ value. 

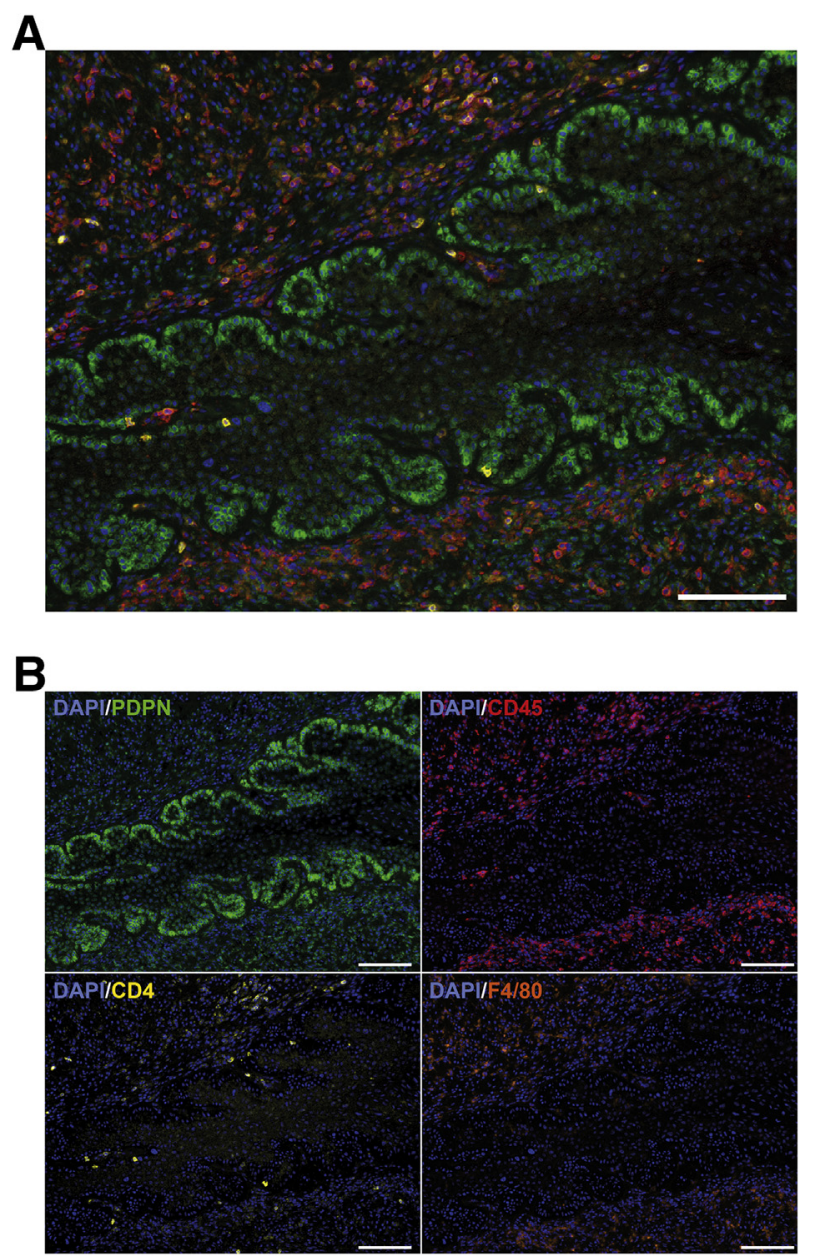

Figure 4 Immune cell infiltration in the tumor stroma and the tumor mass. A: Multiplex immunofluorescence detection of CD45-positive leukocytes in the tumor microenvironment of invasive squamous cell carcinoma (red, CD45; green, PDPN). B: Immunofluoresence detection of PDPN in the tumor invasive front (green), CD45-positive leukocytes in the tumor microenvironment and tumor mass (red), CD4-positive lymphocytes in the tumor stroma and the tumor mass (yellow), and F4/80-positive macrophages in the tumor microenvironment (orange). Scale bars $=50 \mu \mathrm{m}$ (A and $\mathbf{B})$.

squamous cell carcinoma. ${ }^{35,45}$ Within 7 months of age, approximately $90 \%$ of treated females develop invasive cervical cancer (Figure 2A).

Immunostaining of $\mathrm{PDPN}$ on $\mathrm{HPV} / \mathrm{E}_{2}$ cervical $\mathrm{SCC}$ sections revealed that the PDPN expression pattern is identical between human and mouse invasive cervical neoplasia. PDPN is expressed robustly by the invasive cells of the outer layer of $\mathrm{HPV} / \mathrm{E}_{2} \mathrm{SCC}$ but not within the inner tumor mass, and it is only modestly expressed by the outer layer of normal epithelium (Figure 2B). Fluorescence staining intensity of PDPN in SCC was significantly higher than in normal epithelium $(P<0.0001)$ (Figure 2B). Furthermore, the level of PDPN expression at the outer tumor layer significantly correlated with tumor invasiveness $(P<0.05)$ (Figure $2 \mathrm{C}$ ), because the intensity of PDPN staining was highest and well defined in the invasive tumor regions and rather faint and diffuse in less invasive tumor areas.
PDPN-Expressing Cancer Cells Show an InterferonResponsive Gene Signature

To identify the molecular pathways regulating the specific PDPN expression in the invasive cell layers of SCC, gene expression profiling of the invasive tumor front versus the tumor mass was performed in the $\mathrm{HPV} / \mathrm{E}_{2}$ mouse model of cervical carcinogenesis. Cells from the outer layer (tumor front) and the inner layers (tumor mass) of SCC regions were isolated using laser capture microdissection on hematoxylin-stained cervical cryosections of 7-month-old $\mathrm{HPV} / \mathrm{E}_{2}$ females (Figure 3A). Six samples per condition were isolated. PDPN expression at the invasive front of cervical SCC was confirmed after capture on the respective serial sections (Figure 3A). RNA was isolated and processed for microarray analysis on Affymetrix mouse 430_2.0 chips. Principal component analysis revealed the presence of one outlier sample (in the tumor front samples), which was removed from further analysis (Figure 3B). Gene expression profiles of the five remaining tumor front samples were compared with the ones of the six samples isolated from the tumor mass using GeneSpring Software. A total of 209 genes were significantly up-regulated at least twofold $(P<0.05$, one-way analysis of variance) at the invasive tumor front compared with the inner tumor mass (Figure 3C). Among them, PDPN expression was 2.8-fold up-regulated, validating the experimental approach. Gene enrichment analysis of the up-regulated genes using Gene Ontology ${ }^{46}$ revealed that, among many various gene ontology subsets, the type I interferon-mediated signaling pathway and cytokine-mediated signaling pathways were most significantly up-regulated in the PDPN-expressing invasive tumor front (Supplemental Table S1 and Table 1). The up-regulation of PDPN and selected interferon-response genes at the invasive tumor front was subsequently confirmed by quantitative real-time RT-PCR of samples of the PDPN-positive invasive tumor front compared with samples from the PDPN-negative normal epithelium or from the inner tumor mass (Figure 3D). RNA isolated from cells of the outer layer and the inner layers of normal cervical epithelia (isolated from cervical cryosections of nontreated K14-HPV16 age-matched females) was equally processed and analyzed (Figure 3A). No up-regulation of PDPN or the interferon-response genes Ifi47 or $M x 1$ could be observed on normal epithelium (Figure 3D), emphasizing the selective up-regulation of interferon-responses genes at the invasive tumor front.

Interestingly, a strong infiltration of CD45-positive immune cells was observed in the stromal vicinity of invasive SCC areas (Figure 4A). F4/80-positive macrophages localized predominantly to the stroma surrounding the invasive SCC, whereas CD4-positive lymphocytes were also found infiltrating the invasive tumor front (Figure 4B). These data raise the possibility that immune cells of the tumor stroma were the potential source of interferon and inflammatory cytokine signaling. 


\section{A}
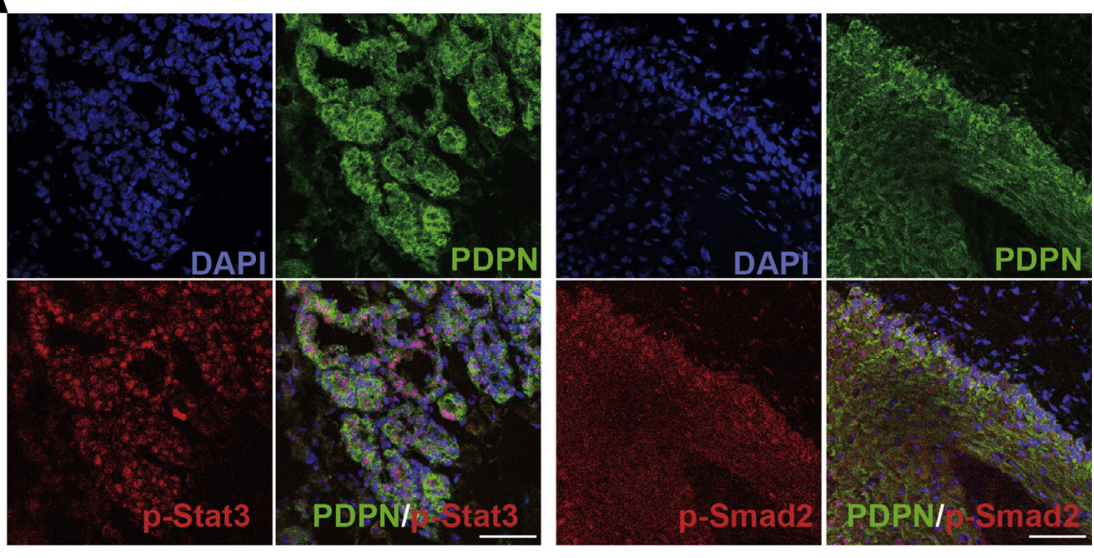

B

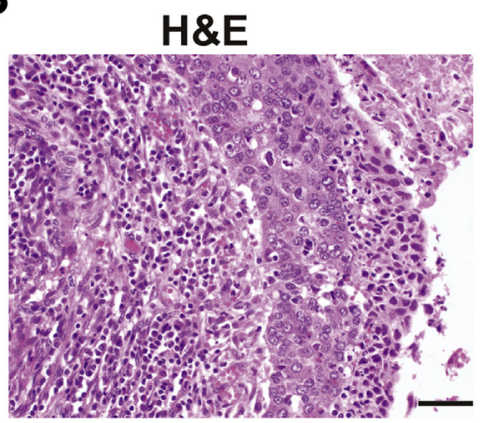

p-STAT1

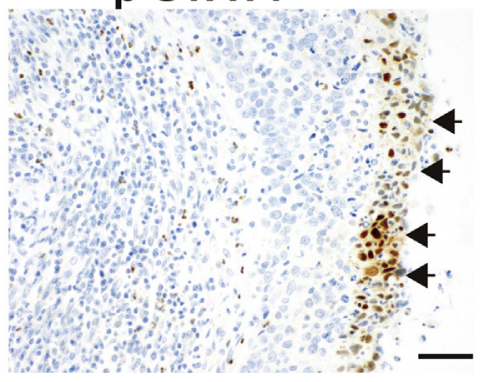

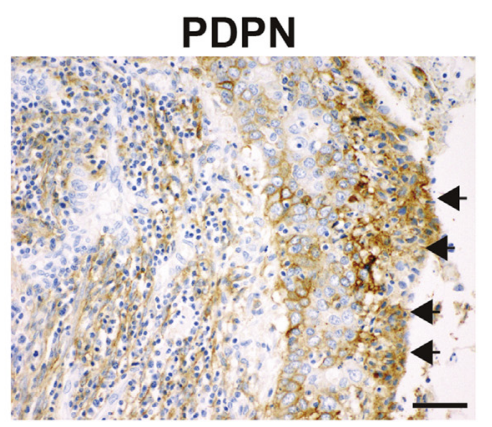

p-SMAD2

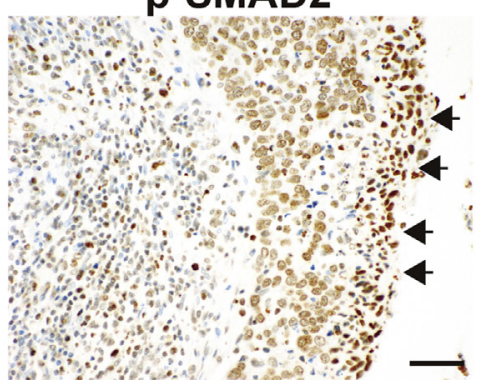

Figure 5 Coexpression of inflammatory cytokine signaling transducers with PDPN in invasive tumor cells. A: Immunofluorescence costaining for phosphorylated (phospho)-Stat3 (red) or phosphoSmad2 (red) with PDPN (green) on histologic sections of cervix squamous cell carcinoma (SCC) from human papillomavirus/estrogen mice. B: Immunohistochemical staining of serial histologic sections from a human cervix SCC with antibodies against phosphorylated (phospho)-STAT1, phospho-SMAD2, and PDPN, as indicated. Representative images are shown. Hematoxylin and eosin (H\&E) staining is shown as orientation and control. Arrows indicate cells of the invasive tumor fronts. $n=10$ (B). Scale bars $=50 \mu \mathrm{m}$ (A and B).
Immunofluorescence staining of histologic sections of SCC of HPV/E $E_{2}$ mice revealed that the signaling effectors of interferons, TGF- $\beta$, and other inflammatory cytokine signaling, phospho-Stat3 and phospho-Smad2, colocalized with PDPN-expressing cells in the invasive tumor areas (Figure 5A). Immunohistochemical staining of histologic sections of cervix SCC of patients revealed that the highest levels of phospho-SMAD2 and phospho-STAT1 also colocalized with PDPN expression within the cells of the invasive fronts (Figure 5B). Although there were also focal nests of higher podoplanin expression that did not exactly colocalize with activated inflammatory signaling, including in cancer-associated fibroblasts (Supplemental Figure S1), inflammatory signaling without podoplanin expression was also detected in cells of the tumor stroma. Together, the results indicate that interferon signaling and interferonresponse genes are activated within the PDPN-positive invasive tumor front. Thus, the data suggest that interferon and other inflammatory cytokines of the tumor stroma may be responsible for PDPN expression and SCC invasion.

\section{Inflammatory Cytokines Induce PDPN Expression in SCC Cells}

On the basis of the correlation between PDPN expression and inflammatory responses in the invasive cells of SCC in vivo, it was assessed whether inflammatory cytokines, including TGF- $\beta$, interferon (IFN)- $\gamma$, IL-6, and tumor necrosis factor- $\alpha$, were able to induce PDPN expression in human epidermal keratinocytes (HEKs) or in various human SCC cell lines, including A431, SCC9, H226, and FaDu cells. Real-time RT-PCR analysis revealed that PDPN mRNA was up-regulated in HEK, A431, and SCC9 cells by treatment with TGF- $\beta$ for 24 hours (Figure $6 \mathrm{~A}$ ). In addition, IFN- $\gamma$ induced PDPN expression in HEK, A431, and SCC9. $\mathrm{FaDu}$ and $\mathrm{H} 226$ cells did not show induced PDPN 
A

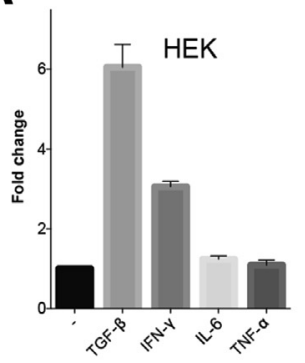

B

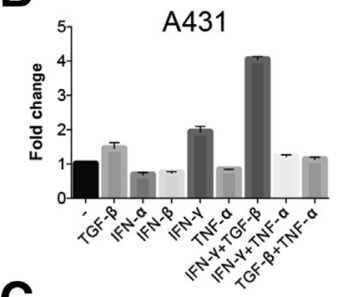

C

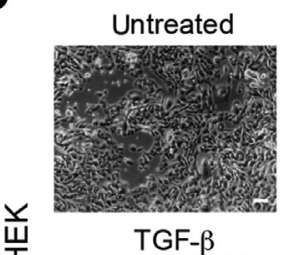

西

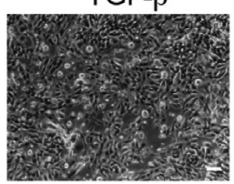

IFN- $y+$ TGF- $\beta$
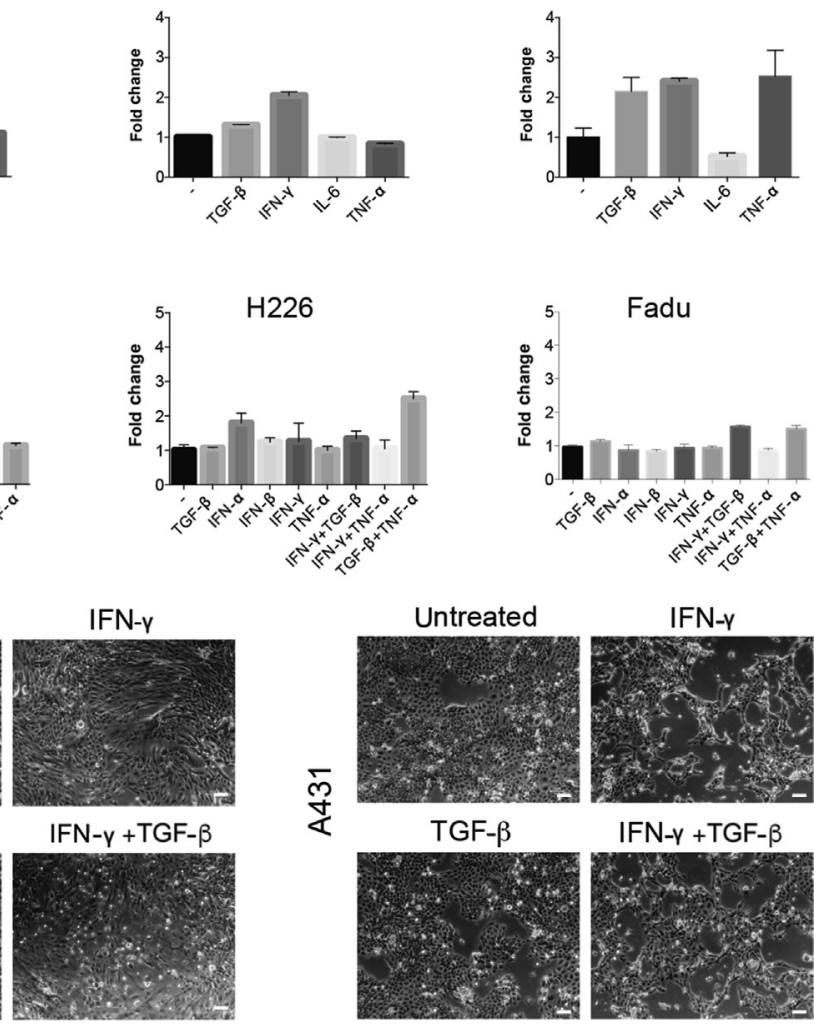

D

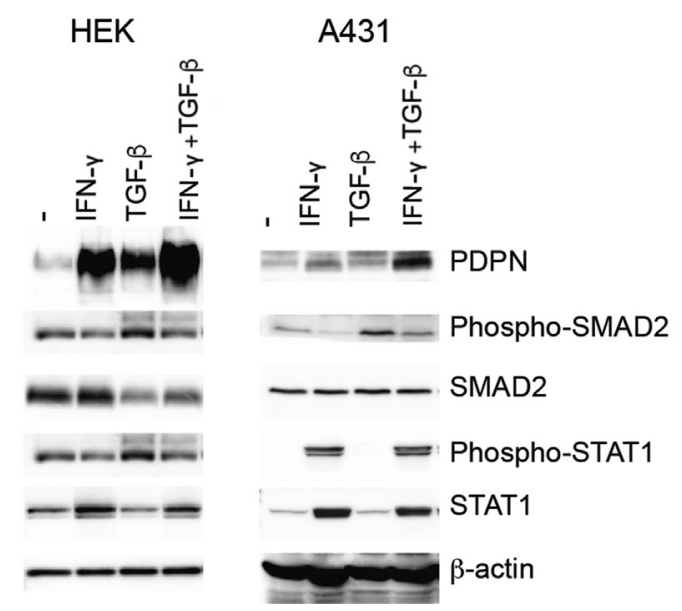

expression by either TGF- $\beta$ or IFN- $\gamma$ alone, yet treatment with both cytokines or with a combination of TGF- $\beta$ with tumor necrosis factor- $\alpha$ induced a slight increase in PDPN expression in these cells (Figure 6B). Hence, more cytokine combinations were examined for their ability to induce PDPN expression in SCC cells. Combination of IFN- $\gamma$ with TGF- $\beta$ showed a synergistic effect in the up-regulation of PDPN in A431 cells over IFN- $\gamma$ or TGF- $\beta$ alone
Figure 6 PDPN induction by inflammatory cytokines in human keratinocyte and squamous cell carcinoma (SCC) cell lines. A: Indicated cells were treated with $2 \mathrm{ng} / \mathrm{mL}$ transforming growth factor (TGF)- $\beta, 50 \mathrm{ng} / \mathrm{mL}$ interferon (IFN) $-\gamma, 10 \mathrm{ng} / \mathrm{mL}$ IL-6, or $10 \mathrm{ng} / \mathrm{mL}$ tumor necrosis factor (TNF)- $\alpha$ for 24 hours, and the expression levels of PDPN were examined by quantitative RT-PCR analysis. B: Dual cytokine treatments of SCC cell lines. Cells were treated with IFN- $\gamma$ plus TGF- $\beta$ or TNF- $\alpha$ or with TGF- $\beta$ plus TNF- $\alpha$ for 24 hours. The expression levels of PDPN were examined by quantitative RTPCR analysis. C: Phase contrast images of human epidermal keratinocyte (HEK) and A431 cells after treatment with TGF- $\beta$ and IFN- $\gamma$ for 48 hours. D: Immunoblotting analysis of PDPN protein expression after treatment of HEK and A431 SCC cell lines with IFN- $\gamma$ and TGF- $\beta$. TGF- $\beta$ stimulation was accompanied by phosphorylation of SMAD2, and IFN- $\gamma$ stimulation was accompanied by phosphorylation of STAT1. Scale bars $=100 \mu \mathrm{m}(\mathrm{C}) .-$, no cytokine added (negative control).
(Figure 6B). In H226, IFN- $\alpha$ alone or a combination of TGF- $\beta$ and tumor necrosis factor- $\alpha$ efficiently induced PDPN expression. Similar individual and combinatorial effects by cytokines were also observed on SCC morphology. In HEK or A431 cells, treatment with IFN- $\gamma$ changed their cell morphology from cobblestone-like epithelial cells to more elongated and spindle-shaped cells, whereas TGF- $\beta$ did not provoke this response (Figure 6C). 
PDPN

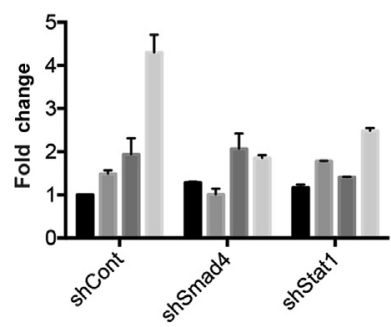

SMAD4

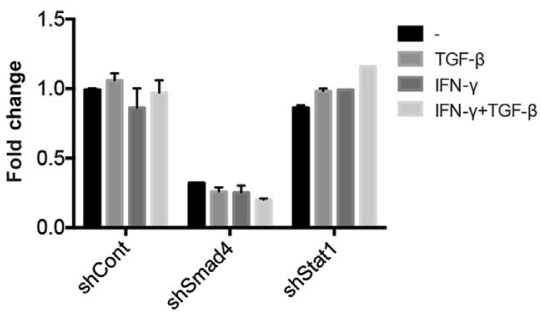

STAT1

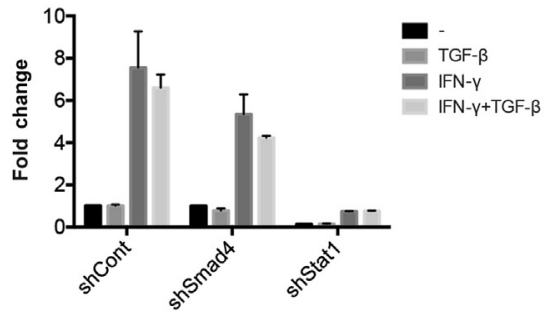

Figure 7 shRNA-mediated ablation of PDPN, STAT1, or SMAD4 represses cytokine-induced PDPN expression. A431 human squamous cell carcinoma cells were stably transfected with constructs expressing a nontargeting shControl (shCont) and shRNAs against SMAD4 (shSmad4) or STAT1 (shStat1) and then treated with transforming growth factor- $\beta$, interferon (IFN)- $\gamma$, or a combination of TGF- $\beta$ and IFN- $\gamma$, and the levels of PDPN, SMAD4, and STAT1 mRNAs were determined by quantitative RT-PCR analysis. - , no cytokine added (negative control).

The combination of IFN- $\gamma$ with TGF- $\beta$ induced a loss of cell-cell contacts and increased cell elongation. Immunoblotting analysis confirmed the up-regulated expression of PDPN by the cytokines (eg, by IFN- $\gamma$ and TGF- $\beta$ in HEK and A431 cells), with higher levels of PDPN on combination of the two cytokines (Figure 6D). Immunoblotting analysis also revealed that TGF- $\beta$ stimulation resulted in phosphorylation of SMAD2, whereas IFN- $\gamma$ treatment induced phosphorylation of STAT1 (Figure 6D). Curiously, IFN- $\gamma$ slightly dampened TGF- $\beta$-mediated SMAD2 phosphorylation in both HEK and A431 cells, although the combination of IFN- $\gamma$ and TGF- $\beta$ synergistically induced PDPN expression.

\section{SMAD and STAT Signaling Pathways Are Required for PDPN Expression}

It was next assessed whether PDPN expression relied on the classic signaling pathways induced by the inflammatory cytokines TGF- $\beta$ and IFN- $\gamma$. A431 cells stably expressing a control shRNA (shCont) or shRNA constructs against SMAD4 or STAT1 (shStat1) were established. Quantitative real-time RT-PCR revealed that the shRNA-mediated knockdown efficiently reduced the expression of SMAD4 and STAT1 mRNA in A431 cells (Figure 7). Treatments of the cells with TGF- $\beta$, IFN- $\gamma$, or a combination thereof showed that the cytokine-induced PDPN expression was attenuated by the shRNA-mediated depletion of SMAD4 and STAT1, respectively (Figure 7). These findings suggest that TGF- $\beta$ and IFN- $\gamma$ induce PDPN expression in SCC cells through SMAD- and STAT-dependent signaling pathways.

\section{Depletion of PDPN or STAT1 Inhibits Tumor Invasion in Vivo}

The functional contribution of interferon and TGF- $\beta$ signaling and PDPN expression to tumor invasion in the A431 xenograft transplantation model of SCC in vivo, which widely recapitulates human $\mathrm{SCC}^{47}$ was next assessed. A431 cells stably transfected with shCont, shRNA constructs against PDPN (shPdpn), shStat1, or shRNA constructs against SMAD4 were injected subcutaneously $\left(1.5 \times 10^{6}\right)$ into the flank of Balb/c nu/nu mice; 17 days later, tumors were collected and histologic sections were prepared and stained with hematoxylin and eosin. A431/shCont or A431/shRNA constructs against SMAD4 tumors showed invasive rough tumor borders, whereas A431/shPdpn or A431/shStat1 tumors showed round
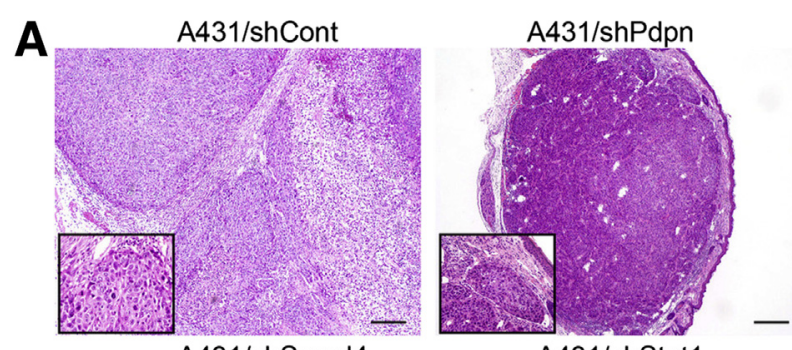

A431/shSmad4
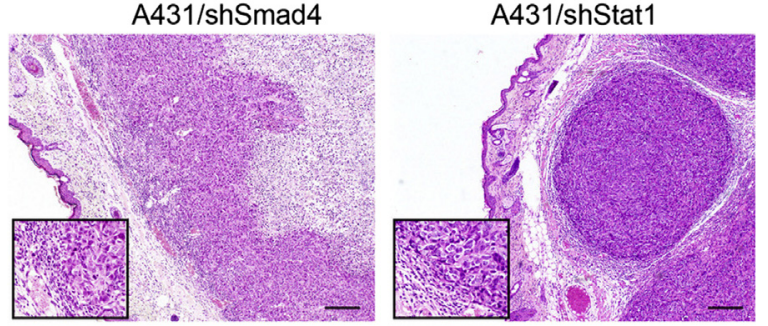

B
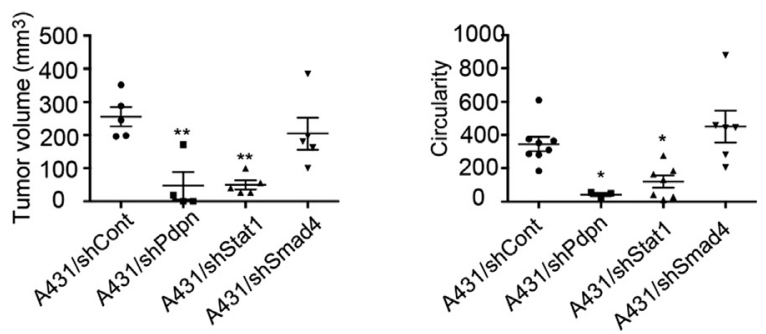

Figure 8 Depletion of PDPN, STAT1, or SMAD4 inhibits A431 squamous cell carcinoma invasion in vivo. A: A total of $1.5 \times 10^{6}$ A431 cells stably expressing nontargeting shRNA (shCont) or shRNAs against PDPN (shPdpn), SMAD4 (shSmad4), or STAT1 (shStat1) were injected subcutaneously into the flanks of immunodeficient mice. Shown are hematoxylin and eosin staining of histologic sections of tumors resected 17 days after implantation. Note the reduced invasive fronts in tumors lacking the expression of PDPN or STAT1 compared with shSMAD4- or shCont-expressing tumors. Insets: Magnification of the areas of interest. B: Tumor volumes (left) and tumor circularity (right) were quantified. Tumor circularity was calculated as $4 \pi$ (surface area/arc length $\left.{ }^{2}\right) . n=8$ shCont and shStat1 (B); $n=4 \operatorname{shPdpn~(B);n=6~shSmad4~}$ (B). ${ }^{*} P<0.05,{ }^{*} P<0.01$. Scale bars $=200 \mu \mathrm{m}(\mathbf{A})$. Original magnification: $\times 20$ (main images); $\times 200$ (insets). 
tumor borders lacking invasive fronts (Figure 8). The volumes of A431/shRNA constructs against SMAD4 tumors were smaller compared with control tumors, but not at a statistically significant level. In contrast, growth of A431/shPdpn and A431/shStat 1 tumors was significantly reduced compared with A431/shCont tumors. Circularity as a quantitative measure of tumor invasion was calculated as $4 \pi$ (surface area/arc length ${ }^{2}$ ), where smaller values indicate more circular and less invasive forms (the value of an exact circle would be 1), whereas higher values represent aberrant invasive forms. As a result, the circularity values of the tumors from A431/shPdpn and A431/shStat1 were significantly smaller than A431/ shCont tumors, indicating that A431/shPdpn and A431/ shStat 1 tumors formed more circular and less invasive tumors.

\section{Discussion}

The results show that, in cervix, SCC of patients or of a mouse model PDPN is expressed in distinct cell layers of the collective invasion fronts. Such expression pattern is rather unique and, thus, begs the question as to how such distinct expression in specific layers of cancer cells is regulated. Laser capture microscopy and microarray gene expression profiling were used to identify the major pathway regulating this distinct expression pattern of PDPN in SCC cells. Notably, the expression of PDPN is induced by inflammatory cytokines most likely secreted by the proinflammatory tumor microenvironment and that interfering with cytokine-mediated signal transduction in SCC cells reduces PDPN expression and, with it, tumor invasion.

These results provide important insights, on one hand because PDPN expression at the tumor invasive front has a prognostic value. ${ }^{10,20,21}$ On the other hand, the finding that a synergistic and/or additive effect of inflammatory cytokines is underlying the induction of SCC invasion indicates a strong contribution of inflammatory events in the tumor microenvironment to SCC invasion and progression. Because no specific up-regulation of the expression of inflammatory cytokines was found in SCC cells at the invasive front, it is likely that immune cells infiltrating the close neighborhood of a growing SCC generate a proinflammatory environment by secreting a cocktail of cytokines. Consistent with this notion, a high number of CD45-positive immune cells were observed in the vicinity of the invading SCC front, in part macrophages and CD4-positive lymphocytes. However, the identity and function of these cells and how they are recruited to the vicinity of the SCC remain to be investigated. Using the K14HPV16 mouse model, Andreu et $\mathrm{al}^{48}$ have previously shown that B cells, humoral immunity, and activating FcgRs are required for establishing chronic inflammatory programs that promote de novo carcinogenesis.

The data indicate that interfering with the cytokine signaling of an inflammatory tumor microenvironment would not only interfere with PDPN expression and with tumor invasion, but also affect many other aspects of inflammation-induced tumor progression. In this context, it would be interesting to learn more about the critical signaling pathways to interfere with, for example, in the A431 xenotransplantation model of human SCC and in the K14-HPV16 mouse model of SCC. Because the signaling pathways acting downstream of PDPN have remained rather elusive, and research into them thus far has not revealed any suitable therapeutic target, a novel strategy to interfere with SCC invasion and progression may be the inhibition of the inflammatory responses triggering PDPN expression itself and thus SCC invasion, a concept demonstrated in this report. Hence, targeting interferon-induced STAT1 signaling may be a valid approach. Janus-activating kinase/ STAT inhibitors are making their way in the clinics, and their use for treatment of SCC progression should be tested.

\section{Acknowledgments}

We thank Prof. Yukinari Kato (Tohoku University) for providing human podoplanin antibodies, NZ-1, LpMab-7, and mouse podoplanin antibody 8F11; Prof. Shosei Kishida (Kagoshima University) and Keith Hoek (University of Zürich) for substantial help with bioinformatics analysis; Kei Sakuma for technical assistance with histology; Pascal Lorentz for excellent support with microscopy; and Helena Antoniadis for technical support.

A.K., V.B., C.M., E.C., and K.T. designed and performed the experiments and analyzed the data; E.G., A.W., M.F., and G.C. designed the experiments and analyzed the data; A.K., V.B., and G.C. wrote the manuscript and obtained final approval by all authors for the submitted version of the manuscript.

\section{Supplemental Data}

Supplemental material for this article can be found at https://doi.org/10.1016/j.ajpath.2018.01.016.

\section{References}

1. Friedl P, Wolf K: Plasticity of cell migration: a multiscale tuning model. J Cell Biol 2010, 188:11-19

2. Haeger A, Wolf K, Zegers MM, Friedl P: Collective cell migration: guidance principles and hierarchies. Trends Cell Biol 2015, 25: $556-566$

3. Yilmaz M, Christofori G: Mechanisms of motility in metastasizing cells. Mol Cancer Res 2010, 8:629-642

4. Shibahara J, Kashima T, Kikuchi Y, Kunita A, Fukayama M: Podoplanin is expressed in subsets of tumors of the central nervous system. Virchows Arch 2006, 448:493-499

5. Ordonez NG: Immunohistochemical diagnosis of epithelioid mesothelioma: an update. Arch Pathol Lab Med 2005, 129:1407-1414

6. Kunita A, Kashima TG, Ohazama A, Grigoriadis AE, Fukayama M: Podoplanin is regulated by AP-1 and promotes platelet aggregation and cell migration in osteosarcoma. Am J Pathol 2011, 179:1041-1049

7. Martin-Villar E, Scholl FG, Gamallo C, Yurrita MM, MunozGuerra M, Cruces J, Quintanilla M: Characterization of human PA2.26 antigen (T1alpha-2, podoplanin), a small membrane mucin 
induced in oral squamous cell carcinomas. Int J Cancer 2005, 113: 899-910

8. Schacht V, Dadras SS, Johnson LA, Jackson DG, Hong YK, Detmar M: Up-regulation of the lymphatic marker podoplanin, a mucin-type transmembrane glycoprotein, in human squamous cell carcinomas and germ cell tumors. Am J Pathol 2005, 166:913-921

9. Wicki A, Lehembre F, Wick N, Hantusch B, Kerjaschki D, Christofori G: Tumor invasion in the absence of epithelialmesenchymal transition: podoplanin-mediated remodeling of the actin cytoskeleton. Cancer Cell 2006, 9:261-272

10. Ugorski M, Dziegiel P, Suchanski J: Podoplanin: a small glycoprotein with many faces. Am J Cancer Res 2016, 6:370-386

11. Martin-Villar E, Megias D, Castel S, Yurrita MM, Vilaro S, Quintanilla M: Podoplanin binds ERM proteins to activate RhoA and promote epithelialmesenchymal transition. J Cell Sci 2006, 119:4541-4553

12. Yonemura S, Hirao M, Doi Y, Takahashi N, Kondo T, Tsukita S, Tsukita S: Ezrin/radixin/moesin (ERM) proteins bind to a positively charged amino acid cluster in the juxta-membrane cytoplasmic domain of CD44, CD43, and ICAM-2. J Cell Biol 1998, 140:885-895

13. Kato Y, Fujita N, Kunita A, Sato S, Kaneko M, Osawa M, Tsuruo T: Molecular identification of Aggrus/T1alpha as a platelet aggregationinducing factor expressed in colorectal tumors. J Biol Chem 2003, 278:51599-51605

14. Nagae M, Morita-Matsumoto K, Kato M, Kaneko MK, Kato Y, Yamaguchi Y: A platform of C-type lectin-like receptor CLEC-2 for binding O-glycosylated podoplanin and nonglycosylated rhodocytin. Structure 2014, 22:1711-1721

15. Schacht V, Ramirez MI, Hong YK, Hirakawa S, Feng D, Harvey N, Williams M, Dvorak AM, Dvorak HF, Oliver G, Detmar M: T1alpha/podoplanin deficiency disrupts normal lymphatic vasculature formation and causes lymphedema. EMBO J 2003, 22: $3546-3556$

16. Uhrin P, Zaujec J, Breuss JM, Olcaydu D, Chrenek P, Stockinger H, Fuertbauer E, Moser M, Haiko P, Fassler R, Alitalo K, Binder BR, Kerjaschki D: Novel function for blood platelets and podoplanin in developmental separation of blood and lymphatic circulation. Blood 2010, 115:3997-4005

17. Honma M, Minami-Hori M, Takahashi H, Iizuka H: Podoplanin expression in wound and hyperproliferative psoriatic epidermis: regulation by TGF-beta and STAT-3 activating cytokines, IFNgamma, IL-6, and IL-22. J Dermatol Sci 2012, 65:134-140

18. Gandarillas A, Scholl FG, Benito N, Gamallo C, Quintanilla M: Induction of PA2.26, a cell-surface antigen expressed by active fibroblasts, in mouse epidermal keratinocytes during carcinogenesis. Mol Carcinog 1997, 20:10-18

19. Suzuki-Inoue K, Osada M, Ozaki Y: Physiologic and pathophysiologic roles of interaction between $\mathrm{C}$-type lectin-like receptor 2 and podoplanin: partners from in utero to adulthood. J Thromb Haemost 2017, 15:219-229

20. Wicki A, Christofori G: The potential role of podoplanin in tumour invasion. Br J Cancer 2007, 96:1-5

21. Renart J, Carrasco-Ramirez P, Fernandez-Munoz B, Martin-Villar E, Montero L, Yurrita MM, Quintanilla M: New insights into the role of podoplanin in epithelial-mesenchymal transition. Int Rev Cell Mol Biol 2015, 317:185-239

22. Durchdewald M, Guinea-Viniegra J, Haag D, Riehl A, Lichter P, Hahn M, Wagner EF, Angel P, Hess J: Podoplanin is a novel fos target gene in skin carcinogenesis. Cancer Res 2008, 68:6877-6883

23. Peterziel H, Muller J, Danner A, Barbus S, Liu HK, Radlwimmer B, Pietsch T, Lichter P, Schutz G, Hess J, Angel P: Expression of podoplanin in human astrocytic brain tumors is controlled by the PI3KAKT-AP-1 signaling pathway and promoter methylation. Neuro Oncol 2012, 14:426-439

24. Scholl FG, Gamallo C, Vilaro S, Quintanilla M: Identification of PA2.26 antigen as a novel cell-surface mucin-type glycoprotein that induces plasma membrane extensions and increased motility in keratinocytes. J Cell Sci 1999, 112:4601-4613
25. Kawase A, Ishii G, Nagai K, Ito T, Nagano T, Murata Y, Hishida T, Nishimura M, Yoshida J, Suzuki K, Ochiai A: Podoplanin expression by cancer associated fibroblasts predicts poor prognosis of lung adenocarcinoma. Int J Cancer 2008, 123:1053-1059

26. Hoshino A, Ishii G, Ito T, Aoyagi K, Ohtaki Y, Nagai K, Sasaki H, Ochiai A: Podoplanin-positive fibroblasts enhance lung adenocarcinoma tumor formation: podoplanin in fibroblast functions for tumor progression. Cancer Res 2011, 71:4769-4779

27. Martin-Villar E, Fernandez-Munoz B, Parsons M, Yurrita MM, Megias D, Perez-Gomez E, Jones GE, Quintanilla M: Podoplanin associates with CD44 to promote directional cell migration. Mol Biol Cell 2010, 21:4387-4399

28. Cueni LN, Detmar M: Galectin-8 interacts with podoplanin and modulates lymphatic endothelial cell functions. Exp Cell Res 2009, 315:1715-1723

29. Krishnan H, Ochoa-Alvarez JA, Shen Y, Nevel E, Lakshminarayanan M, Williams MC, Ramirez MI, Miller WT, Goldberg GS: Serines in the intracellular tail of podoplanin (PDPN) regulate cell motility. J Biol Chem 2013, 288:12215-12221

30. Dumoff KL, Chu CS, Harris EE, Holtz D, Xu X, Zhang PJ, Acs G: Low podoplanin expression in pretreatment biopsy material predicts poor prognosis in advanced-stage squamous cell carcinoma of the uterine cervix treated by primary radiation. Mod Pathol 2006, 19: $708-716$

31. Ito T, Ishii G, Nagai K, Nagano T, Kojika M, Murata Y, Atsumi N, Nishiwaki Y, Miyazaki E, Kumamoto T, Ochiai A: Low podoplanin expression of tumor cells predicts poor prognosis in pathological stage IB squamous cell carcinoma of the lung, tissue microarray analysis of 136 patients using 24 antibodies. Lung Cancer 2009, 63:418-424

32. Kaneko MK, Kato Y, Kitano T, Osawa M: Conservation of a platelet activating domain of Aggrus/podoplanin as a platelet aggregationinducing factor. Gene 2006, 378:52-57

33. Kunita A, Kashima TG, Morishita Y, Fukayama M, Kato Y, Tsuruo T, Fujita N: The platelet aggregation-inducing factor aggrus/podoplanin promotes pulmonary metastasis. Am J Pathol 2007, 170:1337-1347

34. Erpenbeck L, Schon MP: Deadly allies: the fatal interplay between platelets and metastasizing cancer cells. Blood 2010, 115:3427-3436

35. Arbeit JM, Howley PM, Hanahan D: Chronic estrogen-induced cervical and vaginal squamous carcinogenesis in human papillomavirus type 16 transgenic mice. Proc Natl Acad Sci U S A 1996, 93: $2930-2935$

36. Carmona-Saez P, Chagoyen M, Tirado F, Carazo JM, PascualMontano A: GENECODIS: a web-based tool for finding significant concurrent annotations in gene lists. Genome Biol 2007, 8:R3

37. Nogales-Cadenas R, Carmona-Saez P, Vazquez M, Vicente C, Yang X, Tirado F, Carazo JM, Pascual-Montano A: GeneCodis: interpreting gene lists through enrichment analysis and integration of diverse biological information. Nucleic Acids Res 2009, 37: W317-W322

38. Tabas-Madrid D, Nogales-Cadenas R, Pascual-Montano A: GeneCodis3: a nonredundant and modular enrichment analysis tool for functional genomics. Nucleic Acids Res 2012, 40:W478-W483

39. Kato Y, Kaneko MK: A cancer-specific monoclonal antibody recognizes the aberrantly glycosylated podoplanin. Sci Rep 2014, 4:5924

40. Kato Y, Kaneko MK, Kunita A, Ito H, Kameyama A, Ogasawara S, Matsuura N, Hasegawa Y, Suzuki-Inoue K, Inoue O, Ozaki Y, Narimatsu H: Molecular analysis of the pathophysiological binding of the platelet aggregation-inducing factor podoplanin to the C-type lectin-like receptor CLEC-2. Cancer Sci 2008, 99:54-61

41. Kato Y, Kaneko MK, Kuno A, Uchiyama N, Amano K, Chiba Y, Hasegawa Y, Hirabayashi J, Narimatsu H, Mishima K, Osawa M: Inhibition of tumor cell-induced platelet aggregation using a novel anti-podoplanin antibody reacting with its platelet-aggregationstimulating domain. Biochem Biophys Res Commun 2006, 349: 1301-1307 
42. Evangelou E, Kyzas PA, Trikalinos TA: Comparison of the diagnostic accuracy of lymphatic endothelium markers: bayesian approach. Mod Pathol 2005, 18:1490-1497

43. Smith-McCune K, Zhu YH, Hanahan D, Arbeit J: Cross-species comparison of angiogenesis during the premalignant stages of squamous carcinogenesis in the human cervix and K14-HPV16 transgenic mice. Cancer Res 1997, 57:1294-1300

44. Arbeit JM, Munger K, Howley PM, Hanahan D: Progressive squamous epithelial neoplasia in K14-human papillomavirus type 16 transgenic mice. J Virol 1994, 68:4358-4368

45. Giraudo E, Inoue M, Hanahan D: An amino-bisphosphonate targets MMP-9-expressing macrophages and angiogenesis to impair cervical carcinogenesis. J Clin Invest 2004, 114:623-633
46. Ashburner M, Ball CA, Blake JA, Botstein D, Butler H, Cherry JM, Davis AP, Dolinski K, Dwight SS, Eppig JT, Harris MA, Hill DP, Issel-Tarver L, Kasarskis A, Lewis S, Matese JC, Richardson JE, Ringwald M, Rubin GM, Sherlock G; The Gene Ontology Consortium: Gene ontology: tool for the unification of biology. Nat Genet 2000, 25:25-29

47. Atsumi N, Ishii G, Kojima M, Sanada M, Fujii S, Ochiai A: Podoplanin, a novel marker of tumor-initiating cells in human squamous cell carcinoma A431. Biochem Biophys Res Commun 2008, 373:36-41

48. Andreu P, Johansson M, Affara NI, Pucci F, Tan T, Junankar S, Korets L, Lam J, Tawfik D, DeNardo DG, Naldini L, de Visser KE, De Palma M, Coussens LM: FcRgamma activation regulates inflammationassociated squamous carcinogenesis. Cancer Cell 2010, 17:121-134 